\title{
The Requirements for Additional Strength Biowaivers for Immediate Release Solid Oral Dosage Forms in International Pharmaceutical Regulators Programme Participating Regulators and Organisations: Differences and Commonalities
}

\begin{abstract}
Christopher Crane ${ }^{1}$, Gustavo Mendes Lima Santos ${ }^{2}$, Eduardo Agostinho Freitas Fernandes ${ }^{2}$, Craig Simon ${ }^{3}$, Andrew Tam $^{3}$, Diego Gutierrez Triana ${ }^{4}$, Henrike Potthast ${ }^{5}$, Ryosuke Kuribayashi ${ }^{6}$, Yusuke Okada ${ }^{6}$, Aya Myoenzono ${ }^{6}$, Ivan Omar Calderon $^{7}$, Zulema Rodríguez ${ }^{7}$, Ben Jones ${ }^{8}$, Sang Aeh Park ${ }^{9}$, So Young Eum ${ }^{9}$, Clare Rodrigues ${ }^{10}$, Joy van Oudtshoorn ${ }^{11}$, Arno Nolting ${ }^{12}$, Chantal Walther ${ }^{12}$, Matthias S. Roost ${ }^{12}$, Jo-Feng Chi ${ }^{13}$, Wen-Yi Hung ${ }^{13}$, April C. Braddy ${ }^{14}$, Alfredo García-Arieta $^{15}$.
\end{abstract}

1. Therapeutic Goods Administration (TGA). PO Box 100, Woden, ACT 2606, Australia. 2. Agência Nacional de Vigilância Sanitária (ANVISA). SIA Trecho 5, Área Especial 54, Bloco B, Brasília, Brazil. ${ }^{3 .}$ Health Canada, 101 Tunney's Driveway, Ottawa, Ontario K1A 0K9, Canada. 4. Instituto Nacional de Vigilancia de Medicamentos y Alimentos (INVIMA). Carrera 10 No. 64-28. Bogotá. Colombia. ${ }^{5}$ Bundesinstitut für Arzneimittel und Medizinprodukte (BfArM), Kurt-Georg-Kiesinger-Allee 3, 53175 Bonn, Germany. ${ }^{6}$. Pharmaceuticals and Medical Devices Agency (PMDA), Shin-kasumigaseki Building 3-3-2, Kasumigaseki, Chiyoda-ku, Tokyo, Japan. ${ }^{7}$ Comisión Federal para la Protección contra Riesgos Sanitarios (COFEPRIS), Oklahoma No. 14, Colonia Nápoles, Delegación Benito Juárez, 03810, Ciudad de México, Mexico. ${ }^{8}$ New Zealand Medicines and Medical Devices Safety Authority (Medsafe), Ministry of Health, 133 Molesworth Street, Thorndon, Wellington, 6011, New Zealand. 9. Ministry of Food and Drug Safety (MFDS). Osong-eup Heungdeok-gu, Cheongju-si, Chungcheongbuk-do, Republic of Korea. ${ }^{10 .}$ Health $\quad$ Sciences Authority (HSA), Health Products Regulation Group, Pre-Marketing Cluster, Therapeutic Products Branch. 11 Biopolis Way, Helios \#11-01, Singapore 138667, Singapore. ${ }^{11}$. South African Health Products Regulatory Authority (SAHPRA). CSIR, Pretoria, South Africa. ${ }^{12}$. Swissmedic, Schweizerisches Heilmittelinstitut, Hallerstrasse 7, 3012 Bern, Switzerland. 13. Taiwan Food and Drug Administration (TFDA). 161-2, Kunyang St., Nangang, Taipei, 11561 Taiwan, R.O.C. ${ }^{14 .}$ U.S. Department of Health and Human Services, Food and Drug Administration (US FDA), Center for Drug Evaluation and Research, Office of Generic Drugs, 10903 New Hampshire Avenue, Silver Spring, MD 20993, USA. 15. WHO Prequalification of Medicines Programme and Agencia Española de Medicamentos y Productos Sanitarios. C/ Campezo 1. Madrid, 28022, Spain.

Received, September 9, 2019; Revised, September 21, 2019; Accepted, September 24, 2019; Published, September 29, 2019.

\begin{abstract}
In relation to the registration of generic products, waivers of in vivo bioequivalence studies (biowaivers) are considered in three main cases: certain dosage forms for which bioequivalence is self-evident (e.g. intravenous solutions), biowaivers based on the Biopharmaceutics Classification System and biowaivers for additional strengths with respect to the strength for which in vivo bioequivalence has been shown. The objective of this article is to describe the differences and commonalities in biowaivers for additional strengths of immediate release solid oral dosage forms between the participating members of the International Pharmaceutical Regulators Program (IPRP). The requirements are based on five main aspects; the pharmacokinetics of the drug substance, the manufacturing process, the qualitative and quantitative composition of the different strengths, and the comparative dissolution profiles. For the pharmacokinetic aspects, many regulators/agencies have the same requirements. All strengths must be manufactured with the same process, although a few regulators/agencies accept small differences. In relation to the formulation aspects, the data required breaks down into three major approaches based initially on one of those of the EU, the USA or Japan, but there are some differences in these three major approaches with some country specific interpretations. Most regulators/agencies also have the same requirements for the dissolution data, though there are some notable exceptions.
\end{abstract}

\section{INTRODUCTION}

The availability of quality generic products plays an increasingly important role in promoting access to medicines worldwide and in helping to address rising health care costs. This, however, has led to significant pressures on medicines regulatory

\author{
Corresponding Author: Dr Christopher Crane, \\ Pharmaceutical Chemistry Section, Scientific Evaluation \\ Branch, Medicines Regulation Division, Therapeutic Goods \\ Administration, WODEN, ACT, 2606, AUSTRALIA, E-mail: \\ Christopher.crane@health.gov.au
}


authorities charged with the review and approval of these products.

This work was begun by the Bioequivalence Working Group (BEWG) of the International Generic Drug Regulators Programme (IGDRP). This programme was created to promote collaboration and convergence among generic drug regulators in order to address the challenges posed by increasing workloads, globalisation and complexity of scientific issues. The IGDRP has now merged with the International Pharmaceutical Regulators Forum to form the International Pharmaceutical Regulators Programme (IPRP). The work of the BEWG continues as part of the IPRP where it is termed the Bioequivalence Working Group for Generics (BEWGG) (1).

The BEWGG aims to promote greater collaboration, regulatory convergence, and potential mutual reliance on respective bioequivalence assessments in the longer term. This group is composed of the following regulators/agencies: Agência Nacional de Vigilância Sanitária (ANVISA, Brazil), Federal Commission for the Protection against Sanitary Risks (COFEPRIS, Mexico), European Commission / European Medicines Agency (EC / EMA), Health Canada (HC), the Health Sciences Authority (HSA, Singapore), Instituto Nacional de Vigilancia de Medicamentos y Alimentos (INVIMA, Colombia), Medsafe (New Zealand), the South African Health Products Regulatory Authority (SAHPRA), the Ministry of Food and Drug Safety (MFDS, Republic of Korea), the Pharmaceuticals and Medical Devices Agency (PMDA, Japan), Swissmedic (Switzerland), the Taiwan Food and Drug Administration (TFDA), Therapeutic Goods Administration (TGA, Australia), the United States Food and Drug Administration (US FDA), as well as an observer, the World Health Organization (WHO).

In relation to the registration of generic

\footnotetext{
1 For the USFDA, the use of the term "biowaivers" is based on FDA regulations and recommendations for immediate release drug products as specified accordingly. The term"biowaiver" refers to either the decision to waive an in vivo bioequivalence requirement under 21 CFR 320.22 or the decision to accept in vitro bioequivalence data in accordance with 21 CFR 320.24(a).
}

products by the various participating regulators and organisations, waivers of in vivo bioequivalence studies (biowaivers) are considered in three main cases: certain dosage forms for which bioequivalence is self-evident (e.g. intravenous solutions), biowaivers based on the Biopharmaceutics Classification System (BCS) and biowaivers for additional strengths with respect to the strength for which in vivo bioequivalence has been shown ${ }^{1}$.

As part of their ongoing work, this group has previously published the results of a survey of the BCS-based biowaiver requirements of participating members (2) and a survey on the requirements for the acceptance of foreign comparator products (3).

If the conditions of a BCS-based biowaiver are not met, when applying to register a generic product, it is usually required that Applicants submit a bioequivalence study comparing the proposed product (Test product) to a Comparator product to establish that the two products will have equivalent clinical and safety profiles (that is therapeutic equivalence is based on bioequivalence). When there is only one strength of the product, the strength to be used in the bioequivalence study is obvious ${ }^{2}$. However, for a large number of products there is more than one strength. For example, capsules of ziprasidone are available in 4 different strengths $(20,40,60$ and $80 \mathrm{mg}$ ) and fixed-dose combination tablets of atorvastatin and amlodipine may be available in some markets in up to 11 different strengths $(80 \mathrm{mg}$ atorvastatin and $10 \mathrm{mg}$ amlodipine $\{80 / 10\}, 80 / 5$, $40 / 10,40 / 5,40 / 2.5,20 / 10,20 / 5,20 / 2.5,10 / 10$, $10 / 5$ and 10/2.5). The question then becomes if a bioequivalence study (or studies) is needed on each strength or only on certain strengths.

The answer to this question depends on five factors: the pharmacokinetics of the drug substance, the similarity of the manufacturing

\footnotetext{
2 For the majority of cases, products need only be tested in the fasted state or the fed state depending on the dosing instructions. However the USA usually requires both fed and fasted studies and Canada requires fed and fasted studies for Critical Dose Drugs. Further the EU has also published some product-specific bioequivalence guidances that stipulate the performance of BE studies in both the fed and fasted states, e.g. tadalafil and rivaroxaban (4). These occur when it is known that different formulations or strengths of the Reference products have a different food effect. These have been adopted by Australia, and considered by New Zealand, South Africa, Singapore and Switzerland.
} 
process, the qualitative composition, the quantitative composition, and the in vitro dissolution profiles of the different strengths. Thus, it is often possible to register multiple strengths of a generic product series based on the results of bioequivalence obtained for only one strength. In such cases, a biowaiver for the additional strengths is appropriate/considered.

This paper details the requirements that have to be fulfilled to obtain a biowaiver for the additional strengths of immediate release solid oral dosage forms with systemic action for the majority of the regulators and agencies that participate in the IPRP. It first outlines the pharmacokinetic requirements that must be met before such a biowaiver is possible and then describes the in vitro data requirements when it is possible. This is based on a survey conducted within the IPRP BEWGG to identify the differences and similarities between the requirements in the different participating members. This paper does not cover prolonged release or delayed release dosage forms.

\section{MATERIALS AND METHODS}

The IPRP BEWGG conducted a survey on the requirements to waive bioequivalence (BE) studies for additional strengths of immediate release solid oral dosage forms with systemic action with respect to the strength for which in vivo $\mathrm{BE}$ has been shown. This information was obtained from the participating members in the BEWGG and is based on their respective regulatory guidance documents and policies (5-22).

\section{RESULTS}

\section{When is a biowaiver approach possible?}

As mentioned in the Introduction, the approach of allowing a biowaiver for additional strengths depends on the pharmacokinetics of the drug substance, the similarities in the manufacturing process, the qualitative and quantitative compositions of the different strengths, and the in vitro dissolution profiles.

It is important to note that in Australia, Canada, Colombia, the EU, New Zealand, Republic of Korea, Singapore, Switzerland, Taiwan, the USA and the WHO additional strength biowaivers cannot be considered when a Biopharmaceutical Classification System (BCS) biowaiver was granted on the original strength and it is expected that full BCS Biowaiver data are provided for each strength proposed (21-23). Although this aspect is not addressed by guidance in South Africa, the same is expected to apply. This is not the case for Brazil that accepts data for an additional strength biowaiver where the original strength was approved under a BCS biowaiver.

\section{Pharmacokinetic Aspects}

\section{Narrow Therapeutic Index/Range (NTI/NTR).}

The authorities of all participating members accept biowaivers for additional strengths even if the drug substance is considered NTI/NTR. However, Japan and Republic of Korea perform more rigid evaluation for additional strength biowaivers of NTI drugs in some instances. In these regulators/agencies, the deviations from proportionality are classified in different levels. For example, in the case of what is termed a level $\mathrm{C}$ change of NTI drugs, Japan and Republic of Korea accept a biowaiver for additional strengths only if the product has rapid dissolution $(11,14)$. Rapid dissolution means $\geq 85 \%$ of the labelled drug is dissolved in 30 minutes and very rapid $\geq 85 \%$ is dissolved in 15 minutes.

\section{Linearity of pharmacokinetics.}

Not all regulators/agencies have criteria for the linearity of pharmacokinetics, but those that do expect the dose adjusted ratio of the Area Under the Curve (AUC) of the different strengths to be within $\pm 25 \%$ ( $75-133 \%$ when assessed as a ratio). In general this data can be taken from the comparator product prescribing information. Note that this definition of the linearity of pharmacokinetics is specific to this purpose of identifying which strengths require BE studies when there are multiple strengths.

In general, if the pharmacokinetics are linear, all participants require the bioequivalence testing of only one strength when the following criteria are met.

The strength to be tested in the bioequivalence study (or studies) should usually be the highest strength, although when the pharmacokinetics are linear Australia, Brazil, Canada, Colombia, the EU, Japan, Mexico, New Zealand, Republic of Korea, Singapore, South Africa, Switzerland, Taiwan, the USA and the WHO will allow the use of a lower strength if the highest strength cannot be administered to healthy subjects for safety/tolerability reasons (e.g. Aripiprazole Tablets (24)). The USA may also ask for an additional bioequivalence study on a lower or higher strength depending on formulation and/or other safety considerations. 
If the pharmacokinetics are non-linear, Mexico requires a bioequivalence study on at least the highest and lowest strengths and may require bioequivalence studies on the intermediate strengths. Republic of Korea requires a bioequivalence study on the highest strength. Japan has not presently defined the requirements. Waiver considerations are similar for other regulators/agencies as they relate to the following:

- If the pharmacokinetics are greater than proportional over the strengths proposed, a bioequivalence study should be performed on the highest strength (then biowaivers may be considered for the lower strengths based on the other criteria described below). Interestingly, the US FDA has issued a specific guideline for Phenytoin Chewable Tablets where not only the highest strength but the highest therapeutic dose of $300 \mathrm{mg}(6 \times 50 \mathrm{mg})$ has to be tested, which raises the question on whether the highest strength might not be the worst case scenario in the case of more than proportional pharmacokinetics in certain active substances (25).

The EU, Colombia, New Zealand, Singapore, South Africa, Switzerland and WHO will accept the use of a lower strength if the highest strength cannot be administered to healthy subjects for safety/tolerability reasons, but in Australia, Brazil and Republic of Korea (and on a case-by-case approach Canada), where these pharmacokinetics apply it is expected that the bioequivalence study will be performed on the highest strength using patients.

- If the pharmacokinetics are less than proportional over the therapeutic dose range, the studies to be performed depend on other criteria.

If this is due to saturation of absorption, only one bioequivalence study at the lowest strength or a strength in the linear range need be performed (e.g. gabapentin).

However, if this is due to limited solubility, at least two bioequivalence studies should be performed at the lowest and highest strengths proposed. Biowaivers might then be granted for the intermediate strengths based the other criteria described below (e.g. acyclovir).

Finally, a higher dose (that is multiple units of the highest strength) may be administered if the sensitivity of the bioanalytical method is low (though acceptance of this would be very unusual given the analytical techniques available today). This also depends on the pharmacokinetics being linear or more than proportional (i.e. not limited by solubility) and the lack of any tolerability issues.

\section{Manufacturing and Formulation Aspects}

In relation to the formulation aspects, Japan and Republic of Korea have a different approach compared to the other participating members. These are detailed in section 5.f. below.

Aspects for the other participating members are described below.

\section{Manufacturing facility and process.}

All participating members require that the manufacturing process used to manufacture the additional strengths of the products is the same as that used to manufacture the strength tested in the bioequivalence study (or studies).

Apart from the EU, Australia, Brazil, Colombia, New Zealand, South Africa, Switzerland and for applications to the WHO prequalification programme, the site of manufacture of the additional strengths of the product has to be the same as that used to manufacture the strength used in the bioequivalence study. In these seven jurisdictions, a separate site or sites can be used if the method of manufacture is the same and appropriate manufacturing process validation data have been generated. Republic of Korea and Singapore prefer that the site of manufacture of the additional strengths be the same as that of the strength used in the bioequivalence study. However, if different, the same set of requirements mentioned above will apply. Japan and Canada assess on a caseby-case basis.

4. Qualitative composition of the formulations. All participating members require that the additional strengths are the same qualitative formulations as the strength used in the bioequivalence study, though they allow flavours, colours, coatings and inks to be different. An exception to this is that the type of coating cannot be different (e.g. it cannot be changed between sugar-coated and filmcoated). 


\section{Quantitative composition of the formulations}

\section{a. Dose proportionality of the core excipients.}

All jurisdictions accept biowaivers where the cores of the different strengths are in direct scale (i.e. the core formulations are proportional).

b. High potency/low percentage products.

All jurisdictions accept biowaivers where the masses of the core excipients of the additional strengths are all kept at the same mass (i.e. the amount of the active substance is changed but the amount of the excipients is kept constant for all the strengths and the core masses only differ because of the different masses of drug substance used). But the acceptability of this approach depends on the percentage or mass of the drug substance in the core: Australia, Colombia, the EU, New Zealand, Singapore, South Africa, Switzerland and the WHO state the amount of drug substance in the highest strength must be $\leq 5 \%$ of the core mass; whereas Brazil, Mexico, Taiwan and the USA (21) allow for $\leq 10 \%$ of the core mass. In contrast, Mexico states that the amount of drug substance must be $\leq 10 \mathrm{mg}$, which is also acceptable for Colombia and the WHO. In Canada, the change in $\mathrm{w} / \mathrm{w} \%$ is delimited; therefore mathematically this aligns with the case where drug substance comprises approximately $<5 \%$ of the core mass and on a case-by-case approach.

All jurisdictions also accept biowaivers in cases related to the previous scenario where the cores of the additional strengths are all kept at the same mass but the difference in mass of the drug substance is compensated for by a change in the mass of a filler/diluent excipient in order to obtain the same core mass in all the strengths of the product series. Again this is dependent on the percentage or mass of the drug substance in the core. The same criteria apply as explained above.

There are some exceptions to this. For example Australia, the EU, New Zealand and South Africa would allow the amount of an antioxidant to be changed in the same ratio as the amount of the drug substance, and, Switzerland and the USA state other differences in formulation could be accepted with proper justification. Canada accepts changes in the percentage with respect to the total core weight according to the limits defined in their policy, i.e. the total change in excipients does not exceed 5\% (8). Brazil accepts changes in the quantitative composition as long as the total weight of the dosage form remains within $\pm 10 \%$ of the total weight of the strength that was investigated in the bioequivalence study (or studies) and the amount of active substance and one or more excipients are changed. In addition, Brazil $(6,7)$ may accept minor and moderate changes depending on the function of the excipient, and, other changes can also be accepted if they are technically justified.

Regarding the quantitative compositions of the formulations, Japan and Republic of Korea have a different approach compared to the other participating members, and waive bioequivalence studies using a systematic classification and organisation of the contents of the changes in formulation (Level A, B, C, $D$, and $E)(11,14)$. This is detailed in section $\mathrm{f}$. below.

An example of where a different decision would be made in different participating members is given in Table 1 below.

\section{c. Shape of product.}

The USA FDA has published recommendations about the shape of each strength being similar (26). The other participating members have not published requirements on the shape of the product strengths in relation to biowaivers for additional strengths of products, but may raise this during assessment if the size and shape of the generic products are different from those of the Comparator products (especially if the generic products are much larger than the Comparator products).

\section{d. Fixed-dose combination products.}

There are two main types of fixed-dose combination products (FDCs): those where both drug substances are in the same granulate; and those where each drug substance is in a separate layer, whether these layers are stacked, concentric or separate (e.g. separate tablets within a capsule).

- When there is a single granulate, most participating members consider the formulation criteria ( $5 \mathrm{a}$ and $5 \mathrm{~b}$ above) for each drug substance separately and that any other drugs substance can be considered as an additional excipient. For Mexico this is not yet defined and for Colombia and the WHO this is not described. 
Table 1. Example of compositional difference between strengths detailing where it would or would not be accepted for an additional strength biowaiver.

\begin{tabular}{|c|c|c|c|c|c|c|}
\hline Ingredient & $\begin{array}{l}\text { Amount } \\
\text { bio } \\
\text { strength } \\
\text { (mg) }\end{array}$ & $\begin{array}{c}\% \text { of } \\
\text { formulation }\end{array}$ & $\begin{array}{l}\text { Amount other } \\
\text { strength (mg) }\end{array}$ & $\begin{array}{c}\% \text { of } \\
\text { formulation }\end{array}$ & Accepted in & Not accepted $^{b}$ in \\
\hline \multirow{7}{*}{$\begin{array}{l}\text { Active } \\
\text { ingredient } \\
\text { Filler/diluent } \\
\text { Excipient } 2 \\
\text { Excipient } 3 \\
\text { Excipient } 4 \\
\text { Excipient } 5 \\
\text { Total }\end{array}$} & 5.00 & $9.09 \%$ & 1.00 & $1.82 \%$ & $\begin{array}{c}\text { Brazil } \\
\text { Colombia }\end{array}$ & \multirow{7}{*}{$\begin{array}{c}\text { Australia } \\
\text { Canada } \\
\text { EU } \\
\text { New Zealand } \\
\text { Singapore } \\
\text { Switzerland }\end{array}$} \\
\hline & 35.00 & $63.64 \%$ & 39.00 & $70.91 \%$ & Mexico & \\
\hline & 8.00 & $14.55 \%$ & 8.00 & $14.55 \%$ & Japan $^{\mathrm{a}}$ & \\
\hline & 3.00 & $5.45 \%$ & 3.00 & $5.45 \%$ & South Africa & \\
\hline & 3.00 & $5.45 \%$ & 3.00 & $5.45 \%$ & Taiwan & \\
\hline & 1.00 & $1.82 \%$ & 1.00 & $1.82 \%$ & USA & \\
\hline & 55.00 & $100 \%$ & 55.00 & $100 \%$ & $\begin{array}{c}\text { WHO } \\
\text { Republic of } \\
\text { Korea }^{\mathrm{a}}\end{array}$ & \\
\hline
\end{tabular}

a Where other criteria are met, see references 11 and 14 .

b Not accepted as the amount of active substance is $>5 \%(9.09 \%)$ in the highest strength and the excipient difference exceeds $5 \%$.

- When the products are bilayered, Singapore, Taiwan and the USA consider this a single unit for the purposes of the formulation criteria $5 \mathrm{a}$ and $5 \mathrm{~b}$ above. Australia, Canada, the EU, Japan, New Zealand, Republic of Korea and Switzerland consider the layers as two separate units. This criterion is based on the fact that patients may take several units of the individual comparator products simultaneously. For Brazil Colombia, Mexico, South Africa and the WHO this is not described.

The agencies were asked if they could approve generics of all eleven (11) strengths of atorvastatin/amlodipine tablets (see Introduction above) based on a single bioequivalence study performed on the highest strength. Brazil and the USA said yes, this was possible, whereas Mexico said this was not yet defined and the other participating members said no as it was unlikely that formulation criteria $5 \mathrm{a}$ and $5 \mathrm{~b}$ above would be met and therefore they would expect two bioequivalence studies at the extremes (see Bracketing below). It was recognised that in some strengths the 5\% rule might apply. For instance, if the total tablet core weight was $400 \mathrm{mg}$ or higher the strengths containing up to $20 \mathrm{mg}$ of atorvastatin (i.e. 20/10, 20/5, 20/2.5, $10 / 10,10 / 5$ and $10 / 2.5 \mathrm{mg}$ ) could be waived based on a bioequivalence study (or studies) in one strength (or up to 40 $\mathrm{mg}$ of atorvastatin (i.e. 40/10, 40/5,
$40 / 2.5 \mathrm{mg}$ ) if the core weight was $800 \mathrm{mg}$ or higher). However, the strengths containing $80 \mathrm{mg}$ of atorvastatin would require a further bioequivalence study (or studies) (i.e. $80 / 10$ and $80 / 5 \mathrm{mg}$ ) because a tablet of $1,600 \mathrm{mg}$ is too large.

\section{e. Bracketing when the above criteria are not met.}

If the formulation criteria set out in the sections 4 and $5 \mathrm{a}$ to $5 \mathrm{~d}$ above are not met, many participating members will accept a bracketing approach where two bioequivalence studies are needed. These studies should be performed with the strengths that represent the extremes, which are generally the highest and the lowest strengths. However, in the case of fixed dose combination products it may be difficult to identify the extremes since not only the extremes in strength, but also the ratio between the active substances and excipients, and the ratio between the active substances themselves may be considered. In addition, it is expected that the dissolution profiles are similar in all the strengths or the extremes in dissolution rate agree with the extremes in composition. This approach is acceptable in Australia, Canada, Colombia, the EU, New Zealand, Singapore, South Africa, Switzerland, Taiwan and the WHO. It is not acceptable in Brazil, Japan, Republic of Korea, and the USA. Mexico will assess on a case-by-case basis. 


\section{f. Japanese and Republic of Korea regulations for Level of formulation change}

For these two countries, the dissolution requirements associated with an additional strength biowaiver (see below) depend (in part) on the level of formulation change between the strengths. The concept is that a small formulation range should not significantly alter bioavailability. The level of change is generally calculated by comparison to strength used in the BE studies. The level of change should be determined by calculating the differences in content of each excipient $(\% \mathrm{w} / \mathrm{w})$ based on the "function of the excipient and the component," as shown in Table 2. It is important to note that the level will depend on the worst case excipient (e.g. if magnesium stearate differs by more than $0.75 \% \mathrm{w} / \mathrm{w}$, but all other excipients are the same $\% \mathrm{w} / \mathrm{w}$, level $\mathrm{E}$ applies). For Republic of Korea, colouring and fragrance agents are not considered when determining the level change. For Japan, concrete examples of calculation methods are described in the Appendix A and B of Q \& A document (27).

Table 2. Levels of formulation change for immediate release products

\begin{tabular}{|c|c|c|c|c|}
\hline \multirow[b]{2}{*}{ Function of excipient and component } & \multicolumn{4}{|c|}{$\begin{array}{c}\text { Difference in excipient content } \\
\text { compared to BE study strength }(\% \mathrm{w} / \mathrm{w})\end{array}$} \\
\hline & $\mathrm{B}$ & $\mathrm{C}$ & $\mathrm{D}$ & $\mathrm{E}$ \\
\hline \multicolumn{5}{|l|}{ Part : Core } \\
\hline \multicolumn{5}{|l|}{ Disintegrating agents } \\
\hline Starch & $\leqq 3.0$ & $\leqq 6.0$ & $\leqq 9.0$ & $>9.0$ \\
\hline Others & $\leqq 1.0$ & $\leqq 2.0$ & $\leqq 3.0$ & $>3.0$ \\
\hline Binders & $\leqq 0.50$ & $\leqq 1.0$ & $\leqq 1.5$ & $>1.5$ \\
\hline \multicolumn{5}{|l|}{ Lubricants $\cdot$ Polishers } \\
\hline Stearate salts & $\leqq 0.25$ & $\leqq 0.50$ & $\leqq 0.75$ & $>0.75$ \\
\hline Others & $\leqq 1.0$ & $\leqq 2.0$ & $\leqq 3.0$ & $>3.0$ \\
\hline \multicolumn{5}{|l|}{ Fluidizing agents* } \\
\hline Talc & $\leqq 1.0$ & $\leqq 2.0$ & $\leqq 3.0$ & $>3.0$ \\
\hline Others & $\leqq 0.10$ & $\leqq 0.20$ & $\leqq 0.30$ & $>0.30$ \\
\hline Diluting agents & $\leqq 5.0$ & $\leqq 10$ & $\leqq 15$ & $>15$ \\
\hline $\begin{array}{l}\text { Others } \\
\text { (Preservatives, Sweeteners, Stabilisers } \\
\text { etc.) }{ }^{1)}\end{array}$ & $\leqq 1.0$ & $\leqq 2.0$ & $\leqq 3.0$ & $>3.0$ \\
\hline $\begin{array}{l}\text { Sum of absolute values of difference of } \\
\text { content }(\%) \text { of changed components }\end{array}$ & $\leqq 5.0$ & $\leqq 10$ & $\leqq 15$ & $>15$ \\
\hline \multicolumn{5}{|l|}{ Part : Film coating ${ }^{2)}$} \\
\hline $\begin{array}{l}\text { Sum of absolute values of difference of } \\
\text { content }(\%) \text { of changed components in } \\
\text { film coating layer } 1)\end{array}$ & $\leqq 5.0$ & $\leqq 10$ & $\leqq 15$ & $>15$ \\
\hline $\begin{array}{l}\text { Rate of change }(\%) \text { of film coating } \\
\text { weight } / \mathrm{cm}^{2} \text { of surface area of core } \\
\end{array}$ & $\leqq 10$ & $\leqq 20$ & $\leqq 30$ & $>30$ \\
\hline \multicolumn{5}{|l|}{ Part : Sugar coating } \\
\hline $\begin{array}{l}\text { Sum of absolute values of difference of } \\
\text { content }(\%) \text { of changed components in } \\
\text { sugar coating layer }\end{array}$ & $\leqq 5.0$ & $\leqq 10$ & $\leqq 15$ & $>15$ \\
\hline $\begin{array}{l}\text { Rate of change }(\%) \text { of sugar coating } \\
\text { weight } / \mathrm{cm}^{2} \text { of surface area of core }\end{array}$ & $\leqq 10$ & $\leqq 20$ & $\leqq 30$ & $>30$ \\
\hline \multicolumn{5}{|c|}{$\begin{array}{l}\text { Levels of change for excipients categorised as "Others" determined by separate calculations of the differences in } \\
\text { content }(\%) \text { regarding the respective use. Ignore the components for which the composition is described as "trace } \\
\text { use." }\end{array}$} \\
\hline \multicolumn{5}{|c|}{$\begin{array}{l}\text { Most coatings are included (waterproof coating, undercoating, enteric coating, and controlled release coating), except } \\
\text { sugar coating. }\end{array}$} \\
\hline \multicolumn{5}{|c|}{$\begin{array}{l}\text { The shape of the formulation influences the calculation of the surface area of the core. When it is impossible to } \\
\text { calculate the surface area of the shape, the shape of the core is assumed a sphere, and the specific gravity of the core } \\
\text { does not change with the formulation. Note this only applies in Japan and not Republic of Korea) } \\
\text { Fluidizing agents are considered as "Others" in Republic of Korea }\end{array}$} \\
\hline
\end{tabular}


The changes are classified into five levels from A to E. Level A corresponds to the following changes in Japan, whereas Republic of Korea only accepts definition 1.

1. Changes where the ratios of all composition are the same, except components of which composition described as "trace use" $(0.1 \%$ or less of the total amount).

2. Changes of active ingredient within the range not more than $0.5 \%(\mathrm{w} / \mathrm{w})$ where the total weight of formulation is not changed with compensation of the weight change by increasing or reducing diluting agents.

3. Exchange of excipients categorised as "Others" in the same use within the range not more than $1.0 \%(\mathrm{w} / \mathrm{w})$ as sum of absolute values of difference of content $(\% \mathrm{w} / \mathrm{w})$ (e.g. change of sweeteners to other sweeteners).

When the calculation is $\leq$ level $\mathrm{B}$, the change level is $\mathrm{B}$. When the calculation is $>$ level B or $\leq$ level $\mathrm{C}$, the change level is $\mathrm{C}$. When the calculation is $>$ level $\mathrm{C}$ or $\leq$ level $\mathrm{D}$, the change level is $\mathrm{D}$. When the calculation is $>$ level D, the change level is $\mathrm{E}$.

Once the level is determined, other properties (narrow therapeutic index, solubility and rapidity of dissolution) are taken in to account. Under all circumstances, additional strength biowaivers are possible for levels $\mathrm{A}$ and $\mathrm{B}$, but for levels $\mathrm{C}$ and $\mathrm{D}$ a BE study on further strengths may be required, and a BE study is always required on further strengths when the changes are Level E. This is summarised in Table 3 below.

\section{Dissolution data requirements}

All participating members require some comparative dissolution profile data to support biowaivers for additional strengths. This can be broken down into the products that should be compared, the number of units required, the apparatus required, the media required and how the dissolution profiles are compared. Mexico is in the process of deciding on the requirements.

\section{A. Products to be compared.}

In addition to generating dissolution profiles on the different strengths of the test products, Brazil and the USA require that dissolution profiles are generated on the different strengths of the Comparator products. The other participating members do not require the generation of dissolution profiles for the different strengths of the Comparator product, but state that this information can be used as part of a justification for the presence or absence of sink conditions (see point $\mathrm{E}$ below).

Table 3. Levels of formulation changes and required tests for immediate release products in Japan and Republic of Korea.

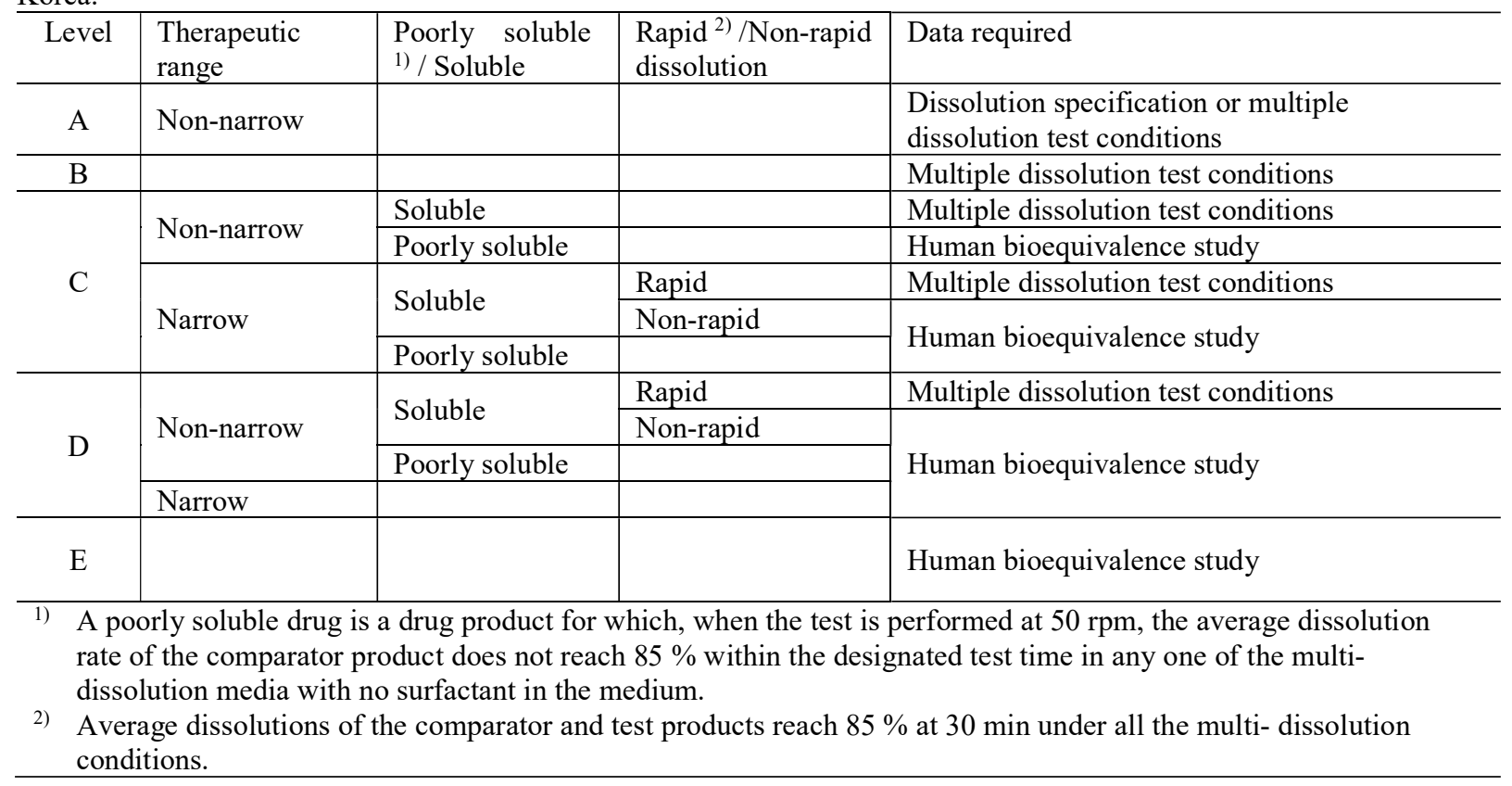


B. Number of units required.

All participating members state that 12 units should be tested for each product and each set of dissolution conditions.

C. Apparatus required.

In general paddle or basket apparatuses are used. Other apparatuses will need justification.

All participating members (apart from Colombia, Mexico and the WHO) expect the paddle speed to be $50 \mathrm{rpm}$. Japan and Republic of Korea do not allow any other paddle speeds and Japan mandates additional data at a paddle speed of $100 \mathrm{rpm}$ for at least one $\mathrm{pH}$ condition when the dissolution at 50 $\mathrm{rpm}$ is not rapid ( $\geq 85 \%$ dissolved in 30 minutes). Whilst other participating members allow for a paddle speed of $75 \mathrm{rpm}$ if this is justified (for instance coning occurs), Japan and Republic of Korea expect a switch to a basket at $100 \mathrm{rpm}$. Colombia and the WHO expect the paddle speed to be $75 \mathrm{rpm}$, but will accept $50 \mathrm{rpm}$.

All participating members (apart from Mexico) expect the basket speed to be 100 rpm. Japan and Republic of Korea do not allow any other basket speeds. Other participating members would allow for the unusually low basket speed of $75 \mathrm{rpm}$ if this is justified.

D. Media Required.

Most participating members require testing at three different $\mathrm{pHs}$ over the physiological $\mathrm{pH}$ range of $\mathrm{pH} 1$ to $\mathrm{pH} 6.8$. Usually the three media are $0.1 \mathrm{~N}$ hydrochloric acid ( $\mathrm{pH} 1.2)$, acetate buffer at $\mathrm{pH} 4.5$ and phosphate buffer at $\mathrm{pH}$ 6.8. Australia, Colombia, the EU, New Zealand, Singapore, South Africa, Switzerland and the WHO require the three $\mathrm{pH}$ media and the quality control medium (if different to the three buffer $\mathrm{pH}$ media described above). Canada would accept testing using either a validated quality control method that has been demonstrated to be discriminating with respect to modest changes in formulation and manufacturing process, or testing with suitable methods in at least three media within the physiological $\mathrm{pH}$ range. In addition, Japan and Republic of Korea require dissolution data in water. However, Brazil and the USA only require testing in the agreed quality control medium. Australia and New Zealand allow some changes to these media if justified.
Japan and Republic of Korea require the use of different media based on the properties of drug substance and products (e.g. products containing acidic drugs, products containing neutral or basic drugs and products containing poorly soluble drugs all require different dissolution media). If the change level is A, Japan and Republic of Korea only require the agreed quality control medium when the strengths are all in direct scale. Therefore, the requirements are simpler than those employed by the majority of the participating members that require multiple $\mathrm{pH}$ buffers and the QC test for excipient compositions similar to those of level A. For changes other than level A, Japan and Republic of Korea require the use of multiple dissolution test conditions.

There are varied requirements about whether a surfactant can be added to the media when the solubility of the drug substance is low. The EU, South Africa and the WHO allow the use of surfactant only in the quality control medium. Brazil and the USA also allow the use of surfactant as they require testing in only the quality control medium. Australia, Canada, Colombia, New Zealand, Singapore, Switzerland and Taiwan, allow this if it is justified which generally requires the generation of data in the media with and without the surfactant (and sometimes with varying amounts of the surfactant to ensure the minimal amount of surfactant is used to achieve reasonable sink conditions and/or sufficient release to allow for assessment of similarity). Republic of Korea allows the addition of surfactants to all dissolution media including the quality control medium, but also requires dissolution profiles in the media without surfactants.

Japan allows the usage of a surfactant ( $\leq$ $0.1 \%$ polysorbate 80 ) only in the case of poorly soluble products, and requires the multiple dissolution media with and without the surfactant.

Table 4 below details the dissolution media expected for the agencies of the various participating members.

E. How to compare the dissolution profiles. All regulators/agencies (except Japan) expect the dissolution profiles of test and Comparator products to be compared using the $f_{2}$ calculation unless the dissolution is very rapid (> $85 \%$ in 15 minutes). Japan adopts $\geq 85 \%$ in 15 minutes. For example see Appendix 1 in reference (11). 
Table 4. Dissolution media to be used for comparative dissolution profiles for an additional strength biowaiver for each participating member jurisdiction.

\begin{tabular}{|c|c|}
\hline Dissolution media to be used & Regulators/agencies where this is acceptable \\
\hline Quality control medium ${ }^{\mathrm{a}}$ only & Brazil, USA, Japan $^{\mathrm{b}}$, Republic of Korea ${ }^{\mathrm{b}}$, Canada ${ }^{\mathrm{c}}$ \\
\hline $\begin{array}{l}\text { Three } \mathrm{pH} \text { buffers over physiological range only (e.g. 1.2, } 4.5 \\
\text { and } 6.8 \text { ) }\end{array}$ & Canada ${ }^{\mathrm{c}}$, Taiwan \\
\hline Three pH buffers over physiological range plus water & Japan $^{\mathrm{d}}$, Republic of Korea ${ }^{\mathrm{d}}$ \\
\hline $\begin{array}{l}\text { Three } \mathrm{pH} \text { buffers over physiological range plus quality } \\
\text { control medium } \\
\text { (e.g. } 1.2,4.5,6.8 \text { and QC) }\end{array}$ & $\begin{array}{c}\text { Australia, EU, Colombia, New Zealand, Singapore, } \\
\text { South Africa, Switzerland, WHO }\end{array}$ \\
\hline $\begin{array}{l}\text { Additional data with non-quality control } \mathrm{pH} \text { buffers } \\
\text { containing a surfactant to demonstrate sink conditions were } \\
\text { not met }\end{array}$ & Australia, Canada, New Zealand \\
\hline $\begin{array}{l}\text { Additional data with non-quality control } \mathrm{pH} \text { buffers } \\
\text { containing a surfactant for poorly soluble products }\end{array}$ & Japan $^{\mathrm{d}}$, Republic of Korea ${ }^{\mathrm{d}}$ \\
\hline
\end{tabular}

a May contain a surfactant

b For Level A cases only, see references 11 and 14.

c Canada allows testing in Quality control medium if the method has been demonstrated to be discriminatory or testing in 3 media within the physiological $\mathrm{pH}$ range with suitable methods.

d See references 11 and 14 .

Japan and Republic of Korea require that two criteria must be met, namely the equivalence of the average dissolution rate and of the individual dissolution variability. For evaluation of the average dissolution rate, Japan and Republic of Korea accept two methods: either of comparing the average dissolution rate between the strengths at some appropriate time points, or the $\mathrm{f}_{2}$ calculation $(11,14)$.

In some cases the dissolution profiles may not be similar due to the lack of sink conditions. In such cases, all regulators/agencies (other than Brazil and the USA) require data to show that sink conditions are not met. This may be done by comparing two different strengths at the same dose (e.g. 2 x $5 \mathrm{mg}$ tablets versus a $10 \mathrm{mg}$ tablet). In addition, Australia, Colombia, the EU, South Africa and the WHO may consider the dissolution profiles of the Comparator product to demonstrate that it also does not give complete dissolution for those strengths. Brazil, Canada, Mexico and the USA may require the data on a case-by-case basis.

\section{DISCUSSION}

This paper shows that the requirements for the additional strength biowaivers for immediate release solid oral dosage forms differ between jurisdictions. These can be classified into three main groups. In Japan and Republic of Korea, the requirements are more complex than for the other jurisdictions due to the classification of the products into several categories with different requirements for the dissolution media to be tested in each category. In contrast, in the EU, Australia, Colombia, New Zealand, South Africa, Singapore, Switzerland and the WHO, the requirements are more straightforward and the same for all products with only three acceptable approaches with respect to excipient composition, and dissolution profile comparison always being required in four dissolution media: the quality control (QC) medium and buffers at $\mathrm{pH} 1.2,4.5$ and 6.8. The most flexible approach is found in the USA and Brazil where the dissolution profiles are compared only in the QC medium and there is more flexibility with respect to excipient composition. Therefore, the requirements for additional strength biowaivers of immediate release solid oral dosage forms is a potential topic for future harmonisation in the International Council for Harmonisation.

In principle, bioequivalence should be demonstrated for all strengths of a generic product versus the corresponding strengths of the comparator product. However, under the assumption that the bioavailabilities of the different strengths of both the comparator (reference) product and generic product (test) are similar (in relative terms), it is possible to demonstrate bioequivalence between one strength of the test and comparator product and extrapolate the safety and efficacy to the additional strengths. The assumption that different strengths of the comparator product exhibit the same 
bioavailability in relative terms is based on the information available in the dossier of the comparator product. The assumption that the bioavailability of the different strengths of the generic product will also be the same is based on the compliance with stipulated in vitro requirements (i.e. same manufacturing process, similar qualitative and quantitative composition and similar dissolution profiles). Conversely, if the different strengths of the comparator product are known to be not bioequivalent (e.g. 5 tablets of 10 $\mathrm{mg}$ are not considered bioequivalent to 1 tablet of $50 \mathrm{mg}$ of the comparator product Dolutegravir (28)), then bioequivalence between the test and comparator product should be demonstrated with more than one strength. Similarly, if the in vitro requirements used to ensure that the different generic strengths exhibit the same bioavailability (in relative terms) are not fulfilled, then bioequivalence should be demonstrated in those strengths failing to comply with these requirements.

The same manufacturing method should be employed for all strengths of the generic product to assume that the different strengths have the same bioavailability in relative terms. Ideally, the strengths should be manufactured using the same equipment or manufacturing plant; however, for some jurisdictions, additional manufacturing plants would also be considered acceptable for all strengths as long as the requirements for the additional sites are acceptable.

In order to ensure that the bioavailability of the additional strengths is similar to the strength used in the bioequivalence study, the qualitative composition of the excipients should be the same. However, differences in non-functional excipients such as colouring and flavouring agents are acceptable given that they are not expected to affect the bioavailability for most products.

Furthermore, the quantitative composition of the additional strengths is also an important factor when determining the acceptability of a biowaiver from conducting bioequivalence studies for additional strengths. For example, EU guidance documents describe three possible approaches regarding differences in the quantitative composition of additional strengths (10).

In the first approach, the amount of excipients in a product core should change in the same proportion as the active pharmaceutical ingredient. For example, if the amount of drug substance in the core of a tablet is doubled then the amount of each excipient in the core of the tablet or the contents of a capsule should also be doubled. In such cases, the formulations are said to be in direct scale and the percentage of each excipient compared to the core mass is the same for each strength.

A second approach can be used for formulations where the amount of active pharmaceutical ingredient is negligible with respect to the amount of excipients. The EU has arbitrarily defined the limit of "negligibility" for the active substance as $5 \%$ of the core weight (10). Other regions (Colombia, Mexico and the WHO) have defined the limit as absolute amount in milligrams (e.g. $10 \mathrm{mg}(9,12,22))$ but this approach is questionable since it depends on the total weight of the tablet core or capsule content. However, as the tablet weight or capsule content is often more than $200 \mathrm{mg}$, the limit of $10 \mathrm{mg}$ may be even more conservative. Other jurisdictions like the USA do not define a limit (21) but rather refer to when the amount of active drug substance in the dosage form is relatively low. The underlying assumption is the drug itself only has a minor contribution to the overall biopharmaceutic product characteristics which are mainly dependent on the excipients and manufacturing processes.

The third approach is a variation of the previous approach and is intended to maintain the same weight for all additional strengths. As a result, the filler (only) can be changed to compensate for the difference in the amount of active substance in the additional strengths of the generic product because its intended function is to maintain the desired weight. Changes in the quantity of the excipients with other functions would not make sense as it would be an indication that the change in the amount of the drug substance has an effect on the performance of the drug product. For example, a change in the amount of the active substance should not require changes in the amount of disintegrant to maintain the same disintegration time and dissolution profile.

The first and third EU approaches are similar to some of the scenarios included in the level A changes in Japan and Republic of Korea.

The USA and Brazil have a more permissive approach in their guidelines $(6,7,21)$, where it is stated that for high-potency drug substances the total weight of the dosage form may remain nearly the same for all strengths (within $\pm 10 \%$ of the total weight of the strength on which an in vivo study was performed) and the change in any strength is obtained by altering the amount of the active ingredient and one or more of the inactive ingredients (excipients). In this case the requirements are not defined as to how much each individual excipient can be changed, since only the total weight of the dosage form is mentioned. In 
order to align the US approach with the EU approach, it would be necessary to limit the change to fillers. In addition, if the excipients are kept constant with only the amount of drug changing, as per the second EU approach, the limit of $10 \%$ is less restrictive than the $5 \%$ 'negligibility limit' of the EU. This value could be even larger if the fillers are changed to compensate for the difference in the amount of drug. In Japan and Republic of Korea, where the excipients can also be changed, the change of the excipients is regulated with welldefined limits as described in Table 2.

Furthermore, even the second and third approaches described by the EU can be considered inappropriate in relation to certain manufacturing technologies or formulations. For example, in the case of solid dispersions (e.g. glimepiride), the amount of drug relative to the amount of excipient responsible for the solid dispersion and maintaining the supersaturation should be fixed (i.e. the same proportion of active drug vs. excipients should be maintained). Therefore, to ensure bioequivalence of all strengths, the different strengths should be developed only with the "proportional" rule, though the current guidance would allow for greater change.

It should be noted that the demonstration of similar dissolution profiles between the additional strengths of the test product and the strength tested in vivo in the bioequivalence study should not necessarily be interpreted as being predictive of the in vivo performance of the test product. QC methods are usually unable to detect differences in bioavailability between different immediate release formulations. Furthermore, the dissolution method is usually developed by the innovator company for the comparator product and its in vivo predictive value in the test formulation has never been investigated. The dissolution profile comparison between different strengths of the test product should be interpreted as in vitro testing that investigates the possibility of performance differences between a series of strengths of the "same" formulation. If differences are observed, the assumption of the similar bioavailability (in relative terms) between strengths cannot be supported. Consequently, a biowaiver for those strengths where dissimilar dissolution profiles are identified would not be considered acceptable.

Some regulators/agencies (see Table 4) require comparative dissolution profiles in several buffered media in order to increase the probability of detecting performance differences between the different strengths. There has to be a balance in the number of dissolution tests required. This balance is a matter for each agency.
Further, it is important to highlight that while surfactants are added to the QC medium, they should not be added to the buffered media because surfactants generally decrease the discriminatory power of the dissolution tests. Similarly, the lowest agitation speed should be kept since higher agitations also decrease the discriminatory power of the dissolution test. It is acknowledged that dissolution may be incomplete for low solubility drugs in some $\mathrm{pH}$ buffers without surfactants. From a regulatory point of view, an incomplete dissolution profile is preferable over an insensitive dissolution test. To avoid artificial differences caused by a different amount of drug substance to be dissolved per vessel in non-sink conditions, the regulators (other than Brazil and the USA) accept demonstration of similar dissolution profiles with the same dose per vessel in order to have the same non-sink conditions. In addition, in Australia, Colombia, the EU, South Africa and the WHO it is also possible to demonstrate that the same limitations relating to sink conditions are observed with the comparator product. However, this approach is not always applicable since test and comparator products can use different excipients or manufacturing technologies that may lead to different in vitro dissolution profiles for products demonstrated to be bioequivalent in vivo. Therefore, these type of justifications are sometimes necessary when dissolution tests other than the QC media are requested. The alternative of using only the QC medium with surfactants for low solubility drugs unable to dissolve completely in the different buffers without surfactants is not preferred by most regulators/agencies. The lack of sink conditions may also be apparent when a surfactant is present if the amount of surfactant is insufficient to dissolve completely the highest strength.

Another critical factor is the particular strength selected for in vivo bioequivalence testing. In most jurisdictions, this decision is based on whether the pharmacokinetics of the drug are considered linear or non-linear. Whilst the EU guideline (10) refers only to AUC for the purposes of defining the linearity of the pharmacokinetics, Cmax can also be affected by differences in the release rate. Thus ideally, Cmax should also be included in the assessment of pharmacokinetic linearity. For example, glimepiride exhibits linear kinetics with respect to AUC but extremely non-linear kinetics with respect to Cmax. As a result, glimepiride would be considered to be a drug with linear pharmacokinetics based on the EU guidance. When AUC is considered for determining pharmacokinetic linearity, the decision is based on 
point estimates lying within a range of 75 to 133 $\%$. Therefore, drugs that have been traditionally considered to have non-linear pharmacokinetics could be considered as linear for the purpose of the selection of the strength to be tested in bioequivalence studies.

Finally, although the same apparatuses are employed for dissolution testing (i.e. paddle and basket), there are differences in the agitation speed and dissolution media to be used, which are far from harmonisation since they are related to the corresponding pharmacopoeias (e.g. the use of 75 rpm with the paddle apparatus is recommended by the International Pharmacopeia) and guidances (e.g. the use of water is required by Japan). Similarly, the methodology to conduct dissolution profile comparisons is still not harmonised. For example, Japan and Republic of Korea differ from the other IPRP BEWGG participating jurisdictions since they accept a comparison based on the average dissolution rate between strengths at select time points in addition to the $f_{2}$ similarity factor. It may be that this last issue, at least, could be harmonised within the ICH M9 guideline on BCSbased biowaivers (29).

\section{CONCLUSIONS}

The requirements for biowaivers for additional strengths of immediate release oral dosage forms that come in a range of product strengths are based on five main aspects: the pharmacokinetics of the drug substance that defines the strength to be tested in the bioequivalence study (or studies), the similarity in the qualitative and quantitative composition between the different strengths, the use of the same manufacturing process and the similarity of the dissolution profiles of the different strengths.

For the pharmacokinetic aspects many regulators/agencies have the same requirements and harmonisation may be achievable. Agreement exists with respect to the use of the same manufacturing process. In relation to the formulation aspects all regulators/agencies agree that only minor justified changes can be made to the qualitative compositions of the different strengths. However, when it comes to the quantitative compositions, the requirements are based on approaches adopted by one of the EU, the USA or Japan, with some further country specific differences and country specific interpretations. In Japan and Republic of Korea, the requirements are based on classifying products into different categories which lead to different requirements in respect to the dissolution media that should be tested. In contrast, the requirements for the EU, Australia, Colombia, New Zealand, South Africa, Singapore, Switzerland and the WHO are the same for all products with similar approaches to excipient composition and that comparative dissolution profiles should be conducted in four dissolution media (the quality control (QC) medium and buffers at $\mathrm{pH} 1.2,4.5$ and 6.8). The most flexible approach is found in the USA and Brazil where the dissolution profiles are compared only in the QC medium and there is more flexibility with respect to excipient composition.

Companies submitting generic applications should be aware of these differences and note that compliance with the strictest requirements from some regulators/agencies and in particular those requirements for composition and dissolution similarity should ensure that the waiver for additional strengths would be accepted in most IPRP member countries and jurisdictions. Finally, we conclude that the requirements for additional strength biowaivers of immediate release solid oral dosage forms would be an excellent choice as a topic for future harmonisation in the International Council for Harmonisation.

\section{REFERENCES}

1. International Pharmaceutical Regulators Programme (IPRP) Bioequivalence Working Group for Generics (BEWGG).

http://www.iprp.global/workinggroup/bioequivalence-generics

2. A Survey of the Regulatory Requirements for BCS-Based Biowaivers for Solid Oral Dosage Forms by Participating Regulators and Organisations of the International Generic Drug Regulators Programme. J. Pharm. Pharm. Sci., 21, 27-37, 2018.

https://journals.library.ualberta.ca/jpps/index.php/ JPPS/article/view/29579

3. A Survey of the Regulatory Requirements for BCSBased Biowaivers for Solid Oral Dosage Forms by Participating Regulators and Organisations of the International Generic Drug Regulators Programme. J. Pharm. Pharm. Sci., 22, 28-36, 2019.

https://journals.library.ualberta.ca/jpps/index.php/J PPS/article/view/30215

4. EMA Product-specific bioequivalence guidance http://www.ema.europa.eu/ema/index.jsp?curl=pa ges/regulation/general/general content 000625.js p\&mid=WC0b01ac0580848f74

5. Therapeutic Goods Administration (Australia). Part 9, Guidance 15 Biopharmaceutic Studies of the Australian Regulatory Guidelines for Prescription Medicines. https://www.tga.gov.au/book/159-justificationnot-submitting-biopharmaceutic-data 
6. Brazilian Health Regulatory Agency (ANVISA). Resolution RDC $n^{\circ}$ 37, 3 August 2011. Provides information about biowaiver and substitution of bioequivalence studies http://portal.anvisa.gov.br/legislacao\#/visualizar/2 $\underline{8739}$

7. Brazilian Health Regulatory Agency (ANVISA). Resolution RDC no 73, 7 April 2016. Provides information about post marketing changes for medicines

http://www20.anvisa.gov.br/coifa/pdf/rdc73.pdf

8. Health Canada. Bioequivalence of Proportional Formulations: Solid Oral Dosage Forms (1996). https://www.canada.ca/en/healthcanada/services/drugs-health-products/drugproducts/applicationssubmissions/policies/bioequivalence-proportionalformulations-solid-oral-dosage-forms.html

9. Instituto Nacional de Vigilancia de Medicamentos y Alimentos (Colombia). Resolución 1124 de 2016. Por la cual se establece la Guía que contiene los criterios y requisitos para el estudio de Biodisponibilidad y Bioequivalencia de medicamentos, se define el listado de los que deben presentarlos y se establecen las condiciones de las Instituciones que los realicen https:/www.invima.gov.co/images/bioequivalenci a.../Resolución\%201124\%20de\%202016.pdf

10. Committee for Medicinal Products for Human USE (CHMP) European Medicines Agency (EMA). CPMP/QWP/EWP/1401/98 Rev.1 Guideline on the Investigation of Bioequivalence http://www.ema.europa.eu/docs/en GB/document library/Scientific guideline/2010/01/WC500070 039.pdf

11. Ministry of Health, Labour and Welfare (MHLW, Japan). Attachment 2 of Division-Notification 0229 No. 10 of the Pharmaceutical and Food Safety Bureau, dated February 29, 2012 Guideline for Bioequivalence Studies for Different Strengths of Oral Solid Dosage Forms.

http://www.nihs.go.jp/drug/beguide(e)/strength/GL-E 120229 ganryo.pdf

12. Federal Commission for the Protection from Sanitary Risks (COFEPRIS, Mexico). General Information guideline.

https://www.gob.mx/cofepris/documentos/docum entos-informativos-de-medicamentos

13. Medsafe (New Zealand). Part 6: Bioequivalence of medicines, Guideline on the Regulation of Therapeutic Products in New Zealand (June 2015). http://www.medsafe.govt.nz/regulatory/Guideline/ GRTPNZ/bioequivalence-of-medicines.pdf

14. Ministry of Food and Drug Safety (Republic of Korea). Standard on Pharmaceutical Equivalence Study; Notification No. 2017-28 (Apr. 19, 2017, Amended)].

http://www.law.go.kr/행정규칙/의약품동등성시 험기준

15. Health Sciences Authority (Singapore). Appendix 10 Product Interchangeability and Biowaiver
Request for Chemical Generic Applications, Guidance on Medicinal Product Registration in Singapore.

https://www.hsa.gov.sg/content/dam/HSA/HPRG/ Western Medicine/Overview Framework Policie s/Guidelines on_Drug_Registration/DR Guide _ $\mathrm{u}$ pdate 2018/A ppendix $\% 2010$ Product $\% \overline{2}$ Interch angeability $\% 20$ and $\% 20$ Biowaiver $\% 20$ Request $\% 2$ 0for $\% 20$ Chemical $\% 20$ Generic $\% 20$ Drug $\% 20$ Appli cations.pdf

16. South African Health Products Regulatory Authority (SAHPRA) previously the Medicines Control Council (MCC). Biostudies guidelines: https://www.sahpra.org.za/documents/61de452d2. 06 Biostudies Jun15 v6.pdf

17. South African Health Products Regulatory Authority (SAHPRA) previously the Medicines Control Council (MCC). Dissolution guidelines: https://www.sahpra.org.za/documents/0ca3cfe72.0 7 Dissolution Jun15 v5.pdf

18. Swissmedic. Guidance document: Authorisation of human medicinal product with known active pharmaceutical substance HMV4.

https://www.swissmedic.ch/dam/swissmedic/en/do kumente/zulassung/zl hmv iv/zl101 $00 \quad 007 \mathrm{~d}$ wl anleitungzulassungvonhumanarzneimittelnmitbeka nn.pdf.download.pdf/ZL101 $00 \quad 007$ e WL\%20Gu idance $\% 20$ document $\% 20$ Authorisation $\% 20$ of $\% 20$ human $\% 20$ medcine $\% 20$ with $\% 20$ known $\% 20$ active \%20pharmaceutical\%20ingredient.pdf

19. Taiwan Food and Drug Administration (TFDA). Regulation of Bioavailability and Bioequivalence Studies, Ministry of Health and Welfare, Taiwan, 2015.

https://law.moj.gov.tw/ENG/LawClass/LawAll.as px?pcode $=$ L0030065

20. Taiwan Food and Drug Administration (TFDA). Announcement Wei-Shu-Yao-Tzu No.0980364304, Additional Strength Biowaiver for Oral Pharmaceutical Product Registration, 2009. http://regulation.cde.org.tw/doc data display?sid= 1903\&doctype 2

21. US Department of Health and Human Services Food and Drug Administration Center for Drug Evaluation and Research. Draft FDA Guidance for Industry - Bioequivalence Studies with Pharmacokinetic Endpoints for Drugs Submitted Under an ANDA (December 2013).

https://www.fda.gov/regulatoryinformation/search-fda-guidancedocuments/bioequivalence-studiespharmacokinetic-endpoints-drugs-submittedunder-abbreviated-new-drug

22. World Health Organization (WHO). Multisource (Generic) Pharmaceutical Products: Guidelines on Registration Requirements to Establish Interchangeability. Republication of Multisource (Generic) Pharmaceutical Products: Guidelines on Registration Requirements to Establish Interchangeability, WHO Technical Report Series, 
No. 992, Annex 7 with a New Appendix 2. WHO Technical Report Series, No. 1003, 2017, Annex 6. http://apps.who.int/medicinedocs/documents/s232 45en/s23245en.pdf

23. Committee for Medicinal Products for Human USE (CHMP) European Medicines Agency (EMA). Q\&A 6.2 Is it possible to accept an "additional strengths biowaiver" when bioequivalence to the reference product has been established with a BCSbased biowaiver?

http://www.ema.europa.eu/ema/index.jsp?curl=pa ges/regulation/q and a/q and a detail 000179.js p\&mid=WC0b01ac0580aff2ec

24. US Department of Health and Human Services, Food and Drug Administration, Center for Drug Evaluation and Research. Draft Guidance on Aripiprazole Tablets (February 2019) https://www.accessdata.fda.gov/drugsatfda docs/p sg/Aripiprazole $\% 20$ Oral $\% 20$ Tablet $\% 20$ NDA $\% 20$ 021436\%20RV\%20Feb\%202019.pdf

25. US Department of Health and Human Services, Food and Drug Administration, Center for Drug Evaluation and Research. Draft Guidance on Phenytoin Chewable Tablets (May 2017) https:/www.accessdata.fda.gov/drugsatfda docs/p sg/Phenytoin oral $\% 20 \% 20$ chewable $\% 20$ tablet A NDA\%20084427 RV03-17.pdf
26. US Department of Health and Human Services Food and Drug Administration Center for Drug Evaluation and Research. Guidance for Industry, Size, Shape, and Other Physical Attributes of Generic Tablets and Capsules, June 2015

https://www.fda.gov/downloads/drugs/guidances/u cm377938.pdf

27. Ministry of Health, Labour and Welfare (MHLW, Japan). Attachment 2 of Clerical-Notification of the Pharmaceutical and Food Safety Bureau, dated February 29, 2012 Guideline for Bioequivalence Studies for Different Strengths of Oral Solid Dosage Forms $Q \& A$. http://www.nihs.go.jp/drug/beguide(e)/strength/QA-E 120229 ganryo.pdf

28. Dolutegravir film-coated tablets $10 \mathrm{mg}, 25 \mathrm{mg}$ and $50 \mathrm{mg}$ product-specific bioequivalence guidance. https://www.ema.europa.eu/en/documents/scientifi c-guideline/dolutegravir-film-coated-tablets-10mg-25-mg-50-mg-product-specificbioequivalence-guidance-first en.pdf

29. International Council For Harmonisation of Technical Requirements For Pharmaceuticals For Human Use, ICH Harmonised Guideline, Biopharmaceutics Classification System-based Biowaivers, M9, https://www.ich.org/fileadmin/Public Web Site/I $\mathrm{CH}$ Products/Guidelines/Multidisciplinary/M9/M 9EWG DraftGuideline Step2 2018 0606.pdf 